\title{
Radiological Image Compression (RIC) Methodologies for Telemedicine
}

\author{
Manvi Mishra, S.S Bedi, Prabhakar Gupta
}

\begin{abstract}
The goal of this review paper is to systematically analyze various Radiological Image Compression (RIC) mechanisms and conclude the optimal technique among them. A brief introduction of image compression reversible and irreversible methodologies are presented and summarized. To improve the classical lossless compression of low efficiency, a way of image lossless compression with high efficiency is offered. The work followed Efficient XML Interchange (EXI) binary xml technology and progressed consequences will be achieved. Moreover enhanced solution for RIC using EXI is likewise mentioned.
\end{abstract}

Keywords: Telemedicine; Radiographs ; Reversible Compression; Irreversible Compression

\section{INTRODUCTION}

Telemedicine means "Medicine at Distance", is achieved by the transfer of electronic patient records to remote medical centre for diagnosing thus on give consultation by doctors placed in geographically numerous locations and obtain the health status of their essential patients [1]. In such applications, health information of patients such as previous examinations result, lab test results, medical images etc collected in digitized type will be simply transmitted over net-work. Purpose of telemedicine is to reinforce provision to a wider population. Due to its huge size, it requires lots of storage space in its raw state and transmission of it necessitates large bandwidth and transmission time as well as cost. Thus there is a need of medical data to be compressed before transmission or storage. To meet the demand for prime speed transmission of pictures in effective image storage and remote treatment, the economical RIC is important to confirm quality of service. Technically all image data is compressed into two groups as reversible (lossless) and irreversible (lossy) [2]. Some data is lost within the irreversible compression. but several applications like satellite image process and medicine imaging cannot afford any losses in their information and area unit usually compressed victimization lossless compression ways within which solely few vital coefficients got to be encoded whereas majority of insignificant coefficients will be removed while not considerably moving the standard of reconstructed image. Content quality in these applications cannot be

Revised Version Manuscript Received on September 16, 2019.

Manvi Mishra, Research Scholar Department of CSE, Dr APJ Abdul

Kalam Technical University, Lucknow, Uttar Pradesh, India

(Email: manvimishra@yahoo.com)

S.S Bedi, Assistant Professor Department of CSE \& IT, IET MJPRU Bareilly, Uttar Pradesh, India

(Email: erbedi @yahoo.com)

Prabhakar Gupta, Professor Department of CSE, SRMSCET Bareilly, Uttar Pradesh, India

(Email: prama63@yahoo.com) compromised because it considerations vital information. Such sectors demand cost-efficient however high-quality information storage and retrieval. Image transformation, division and secret writing these area unit the fundamental building block of compression method. Image transformation is lossless step in which image is transformed from gray scale values to other domain coefficient. Quantization is done to reduce the precision and to achieve higher compression ratio. The defect of quantization is that it is a lossy operation, which can result into loss of preciseness and irretrievable distortion. After [3] During this article, we present, discuss, classify and compare completely different RIC schemes that offer solutions for large image transfer.

The remainder of this paper is organized as follows. Introduction regarding RIC is given in section1. Background of compression techniques is provided in section 2. Review of literature within which varied schemes of RIC is mentioned in section 3. The Section 4 describe increased solution for RIC victimization EXI. Results and discussion is made public in section 5. Likewise Conclusion and Future work is discussed in Section 6.

\section{TAXONOMY OF IMAGE COMPRESSION TECHNIQUES}

With the increasing growth of technology and entrance into the digital age, numerous reversible and irreversible RIC techniques are exist and depicted in figure 1. Reversible techniques are Predictive and Entropy coding. Example of predictive coding is Differential Pulse Code Modulation (DPCM). Predictive coding operates by reducing the inter pixel redundancy of near located pixels. This type of coding extracts and stores only the new information within the new component. This information is defined by the difference of the pixel and the predicted value of that pixel. The prediction relies on the famous values of the closely settled pixels. 


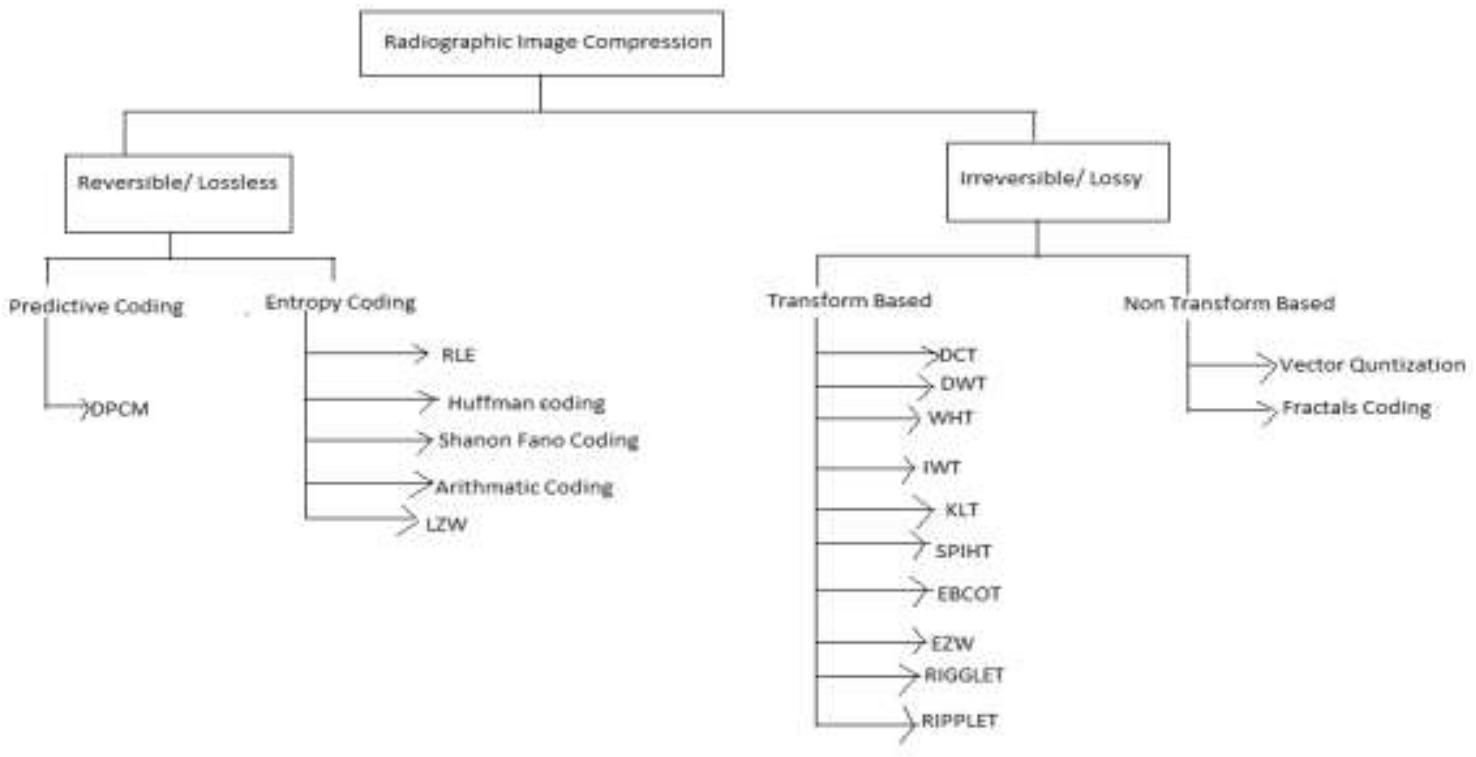

Figure1: Taxonomy of Radiological Image Compression (RIC)

Entropy coding algorithms assign code words to the corresponding measure coefficients in keeping with the likelihood of the measure coefficients. It is created and assigns a prefix code to each distinctive image that happens among the input. These entropy encoders then compress information by substitution each fixed-length input image by the corresponding variable-length codeword. The advantage of exploitation Huffman

or Shannon-Fano before transmission of an information signal is that the information measure accessible is used a lot of with efficiency because of the common bit rate being reduced by the coding method. One disadvantage of the Huffman code is that we have a tendency to cannot begin distribution code bits till the wholecombination method is completed. But the Shannon-Fano code does not invariably offer results pretty much as good as Huffman. [4] it's entropy committal to writing formula wide utilized in the most recent image Huffman coding. In arithmetic encoding, we have a tendency to treat the entire input file as one image and notice the corresponding codeword for it. Thus, the committal to writing potency of the arithmetic code is best. [4 ] Run-length encryption (RLE) RLE may be a type of lossless compression that takes redundant strings and stores them jointly unit. Let there square measure fifteen white pixels and thirteen red pixels. Normally, the info for it'd be written asWWWWWWWWWWWWWWRRRRRRRRRRRRR, with W showing the white component and $\mathrm{R}$ the red component. Run length would place the info as $15 \mathrm{~W}$ and 13R. abundant smaller and easier whereas still keepingthe info timeless. Lempel-Ziv-Welch (LZW) formula is that the following: because the input file is being processed, dictionary keeps a correspondence between the longest encountered words and a list of code values. The words are replaced by their corresponding codes so the file is compressed. Therefore, the potency of the formula will increase because the variety of long, repetitive words within the input file will increase.LZW needs no previous information concerning the input file stream as compare to huffman. [6]. Irreversible and rework primarily based techniques are: Discrete Cosine Transform (DCT), Discrete wavelet Transforms (DWT), Integer Wavelet Transform (IWT), Set Partitioning in Hierarchy Trees (SPIHT), Embedded Zerotree Wavelet (EZW) , Embedded Block Coding with Optimized Truncation of the embedded bit-streams (EBCOT), Ridgelet transform, Ripplet Transform. DCT primarily based compression like JPEG performs alright at moderate bit rates; but, at higher compression ratio, the standard of the image degrades [9] DWT famous methodology for image compression. JPEG-2000 is also based upon DWT. Wavelet decomposes a symptom into a group of basis functions which are called as wavelets. DWT is economical and versatile technique for sub band decomposition of signals. SPIHT exploits the inherent similarities across the subbands during a wavelet decomposition of an image. It encodes the foremost necessary wave rework coefficients

initial, associate degreed transmits the bits so an progressively refined copy of the initial image is obtained more and more. [9] Embedded Zerotree wave (EZW) compression rule, having the property that the bits within the bit stream are generated so as of importance, yielding a completely embedded code. The embedded code represents a sequence of binary choices that distinguish a picture from the "null" image. EZW provide good compression results as compare to available methods Embedded Block secret writing with Optimized Truncation of the embedded bit-streams (EBCOT) EBCOT has been chosen because the framework for the evolving JPEG2000 commonplace .It manufacture a bit-stream with an expensive feature set, as well as resolution and SNR quantifiability beside a random access property. Ripplet transform break the inherent limitations of wavelet. It is a better dimensional generalization of ripple remodelcapable of

Published By: 
representing pictures or 2 dimensional signals at completely different scales and different directions. the same as curvelet, ripplet is additionally optimum for representing objects with C2 singularities. Thus, edges inside pictures have a thin illustration in ripplet area. Ripplet generalizes curvelet by adding two vital parameters i.e. support $\mathrm{c}$ and degree $d$. The introduction of support $c$ and degree $d$ provides property capability of representing singularities on every which way formedcurves [8] Ridgelet transform-Candes and Donoho, 1999 was introduced as a brand new multi scale illustration for functions on continuous areas that are far from discontinuities on lines. However, a disadvantage with the ridgelet transform is that its basis functions usually have larger support than those of the wavelet transform. As ridgelet rework makes speedy progress in reconstructing the image when the primary few coefficients on the other hand slows down signicantly; These non transform based ways are vector quantization and fractal coding .In vector quantization the information values are diagrammatic, in the form of arrays. In vector quantization compression may be lossy or lossless depending upon choice. For every situation the picture is beginning partitioned into littler quadratic $n$ by $n$ areas. The area will at that point be viewed as a posting or vector with $n * n$ values. One lossy way of weight these is by using a code book. Each picture area is then contrasted with the code book vectors. Since the possibility of finding the exact same vector inside the code book is amazingly thin, the nearest vector is picked. A packed information stream only comprises of the images bearing on one code book passage. Pattern Compression is lossy procedure, created by chief heavenly messenger Barnsley and his partners Since the chance of finding the precise same vector within the code book is extremely slim, the nearest vector is chosen [7].Fractal technique has the advantage of quicker decompression speed, having done most of the computation throughout the compression step, whereas giving equal or higher compression ratio. However, fractal compression is not free from its limitations
Table I. Disparate RIC Schemes

\begin{tabular}{|c|c|c|}
\hline MECHANISMS & $\begin{array}{l}\text { Nature of } \\
\text { Image }\end{array}$ & Type \\
\hline DCT & Irreversible & Transform based \\
\hline DWT & Irreversible & Transform based \\
\hline IWT & Irreversible & Transform based \\
\hline WHT & Irreversible & Transform based \\
\hline KLT & Irreversible & Transform based \\
\hline SPIHT & Irreversible & Transform based \\
\hline EBCOT & Irreversible & Transform based \\
\hline EZW & Irreversible & Transform based \\
\hline VQ & Irreversible & Non Transform based \\
\hline Fractal & Irreversible & Non Transform based \\
\hline RLE & Reversible & Non Transform based \\
\hline Huffman Coding & Reversible & Non Transform based \\
\hline Shanon fano Coding & Reversible & Non Transform based \\
\hline Arithmatic Coding & Reversible & Non Transform based \\
\hline JPEG2K & $\begin{array}{l}\text { Reversible, } \\
\text { Irreversible }\end{array}$ & Non Transform based \\
\hline Predictive Coding & Reversible & Non Transform based \\
\hline LZW & Reversible & Dictionary based \\
\hline
\end{tabular}

\section{RADIOLOGICAL IMAGE COMPRESSION METHODOLOGIES (RICM)}

The approach adopted for critical review of research works. Table I. summarizes the reviewed works done by various scientists and researchers on RIC with their critical analysis.

1991, Majid Rabbani reviewed lossless and lossy image compression techniques. DCT along with DPCM is used but DCT need large computational resources and presents blocking artifacts and contour effects in reproduced picture [10]. In 2001, Po-chyisu proposed integrated approach to image watermarking and JPEG2K compression. EBCOT is used [11]. In JPEG2K shape information of VOI needs to be encoded and if arbitrary VOI shape is selected then coding will consume more no of bits which reduces overall efficiency. Salih Burak Gokturk et. al [12] in 2001 discussed hybrid model of lossless medical compression in ROI region and really high rate lossy compression in non ROI region. This scheme results only $2.5 \%$ compression rate as compare to transform based coding. In 2002, Bradley J Erickson M.D. et al.[13] outlined importance of lossy compression of medical images. Result shows that wavelet compression is better than JPEG for radiographs but wavelet transform does not handle curve discontinuities well. In 2006, Rajasvaran Logeswaran et. al.[14] presented model primarily based compression for medical pictures keep in DIACOM format. Biliary track in MRCP pictures is illustrated and important gain in compression rate springs with models like Hierarchy Plot, Hierarchy Hierarchy Growing and Segment growing but 
DIACOM format can't be directly stored through outcome of Hierarchy plot Kamrul Hasan Talukder et al. [15] discussed Haar Wavelet based methodology for image compression and its quality measurement in 2007 but Haar Wavelet is discontinuous and doesn't approximate continuous signal well. In 2009, Lihong Zhao proposed integration approach of medical image compression using combination of IWT and DPCM [16]. Simulation results shows method is easy and useful. Still IWT is weak in smooth curve areas. D. Maheshwari et al. [17] in 2010 outlined layer based compound image compression methodology which uses XML for foreground layer and JPEG for background layer compression. The biggest problem is that XML files are verbose. In 2011, M P Bakuline et al. outlined image compression by encoding of low entropy source coding [18]. Here comparison between JPEG-LS and Entropy source coding is done and concluded that proposed method is better than JPEG-LS but it has less compression ratio as compare to wavelet. In 2011, Nik Shahidah [19] proposed medical image compression predicting wavelet based algorithm .It produce efficient coefficient that are thresholded but DWT coefficient oscillate at zero crossing. In 2012, According to Sujitha Juliet Devaraj proposed 3D IWT and 3D- EBCOT techniqueto the reconstructed medical image slices with Volumn of interest [20]. Result shows improvement in 3D object obtained from reconstructed 3D Images but smooth curve edge creates hindrances in IWT. V.K. Bairagi et al. [21] in 2013 discussed role of transform in image compression .Comparative study among KLT, DCT,WHT,DCT,DWT and IWT transformation is presented and concluded that there is a need of special transform with high compression, less entropy and less complexity. In 2013, Sujitha Juliet [22] discussed medical image compression victimization Ripplet Transform to represent singularities along randomly formed curve and SPIHT to encode coefficient. The given method attains high PSNR and CR as compare to other method but when images are transferred over unreliable network SPIHT algorithm perform poorly. In 2014, Abdul Khader Jilani Saudagar et. al. [23] discussed biomedical image compression approach with Ridgelet transformation using modified neuro modeling. The given technique i.e. combination of ridgelet transformation with hybrid neural network produce better result as compare to JPEG2K .Since outcome produce grayscale images, the color component is not given much value and ridgelet might be ineffective where edges are curved rather than being straight line. In 2014, M. Moorthi et al [24] elaborated integration model which uses separation of ROI and non ROI region where ROI is compressed using Curvelet transform, DPCM and non ROI is compressed using IWT, SPIHT followed by adaptive arithmetic encoding. After fusion results obtained .Performance is degraded in IWT when edges are smooth curve. In 2015, Sujitha Juliet et al [25] proposed novel method for medical image compression using geometrical regularity of image structure. It consists of sparse representation approach along with DWT. It provides significant improvement in compression but DWT lacks directional selectivity. V. K. Bairagi [26], 2015 outlined Symmetry based bio medical image compression SIFT( Scale invariant feature transform)is applied and Huffman coding is used for source encoding. It provides lossless image compression but applied Huffman coding is slow and uses variable length codeword. In 2015, Sujitha Juliet et al. [27] discussed projection based medical image compression which take advantage of random transform and SPIHT algorithm for encoding. Experimental result obtained demonstrate that it provide enhance performance compare with traditional methods but SPIHT are state dependent so susceptible to bit error. Missing or adding extra bit destroying the decoding image. In 2015, Manpreet Kaur and Vikas Wasson [28] elaborated ROI based compression for medical images. Fractal lossy compression for non ROI image and context tree weighting lossless for non ROI part of an image have been projected. Here only three parameters low MSE, high PSNR and CR is evaluated as compare with IWT. In 2015, Emmanuel B.S.et al.[29] discussed fingerprint image compression via three steps-DWT Coefficient transformation, Non Uniform quantization and Arithmatic Entropy encoding. Result shows 20:1 CR over existing compression algorithm but limitation in DWT is that it is not Shift invariant. In 2015, Gram Badshah et al. [30] focused on watermarking of ultrasound radiological images with LZW lossless compression. It performs better result but LZW create entries in dictionary that may never be used.

From the above survey it is observed that several general purpose compression tools have been proposed without satisfactory results so there is a need of an innovative reversible RIC methodology EXI for high speed transmission of radiographs with less CR, low entropy, low MSE, high energy compaction and fewer process complexness whereas protective the diagnostic integrity. Disparate RIC Schemes are tabulated in table II 
Table I .Comparison among various radiological image compression methods

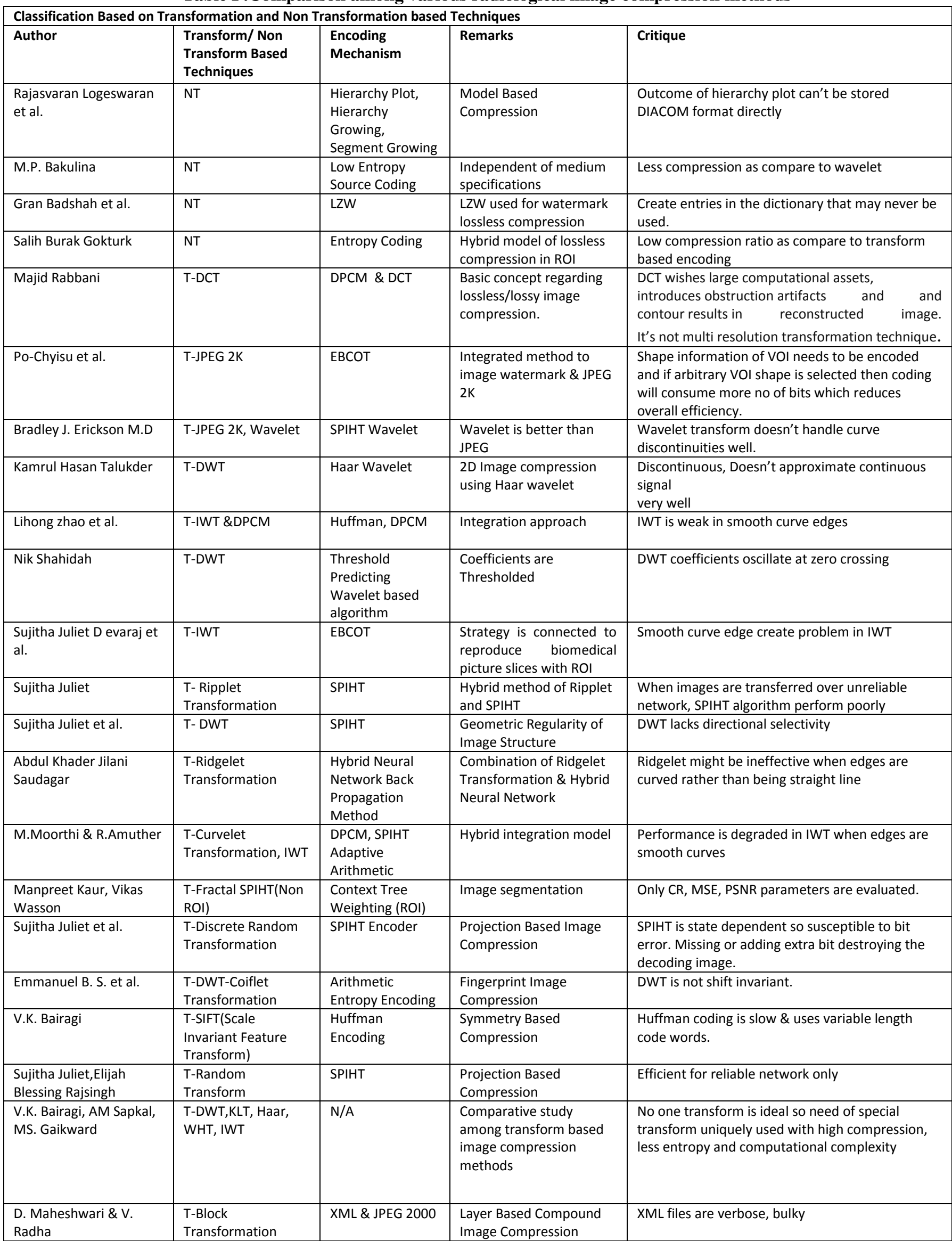




\section{OBSERVATIONS}

From the aforesaid survey it is observed that non transform based radiological image compression methodologies are not very efficient as compare to transform based mechanism. Entropy Coding, dictionary technique such as LZW and model based like hierarchy ( Plot/ Growing), segment growing methods are evaluated in terms of compression ratio, and outcome depicted less compression. Further transform based techniques like DCT, wavelet (DWT, IWT, Haar, WHT) degrades performance when images are transferred in unreliable network and possess curve discontinuities with heavy computational resources. At last transform based secure picture compression using $\mathrm{xml}$ is analyzed. Study reflects that many medical agencies have been adopted cloud computing which is based on XML methodology for storing and transmitting radiological images.XML is basis and prime required component for cloud web services but disadvantages of XML is that it is redundant and verbose.

To avoid aforesaid limitation, binary XML mechanism namely EXI is analyzed and examined. Outcome reveals more improved results achieved in terms of compactness and security of radiological image transmission and storage applied in telemedicine.

\section{RECOMMENDATIONS}

After investigating various technique it is recommended that EXI based compression is the optimum solution for radiological image transmission and storage.EXI offer general, platform neutral, universal acceptable solution for radiographs transmission in very compact form.

\section{REFERENCES}

1 Int' J of Computer Assisted Radiology and Surgery CARS (2006) 1:119-135 E-Health and Telemedicine, Digital medicine in the virtual hospital of the future, G. Graschew Æ T.A. Roelofs, 2000,

2 Usability of irreversible image compression in radiological imaging.A position paper by the European Society of Radiology (ESR), insights imaging ,springer,Jan 2011,pp 103-115

3 Frank Y. Shih et al., Robust watermarking and compression for medical images based on genetic algorithms", Elsvier Int' J .Information Sciences 175(2005),pp. 200-216

4 Michael G. Strintzis, "A review of compression methods for medical images in PACS" Int' $J$ of medical Informatics 52 , Elsevier, 1998, pp 159-165

5 http://www.math.psu.edu/tseng/class/Fractals.html--fract alcoding e Notes are Based on (or use material from): 1. J. M. Shapiro, "Embedded Image Coding Using Zerotrees of Wavelet Coefficients," IEEE Trans. on Signal Processing, Vol. 41, No. 12, pp. 3445 - 3462, Dec. 1993.

6 Sujitha Juliet, Elijah Blessing Rajsingh et al, “ A novel medical image compression using Ripplet transform" Int'J Real Time Image proc,springer, 12 July 2013

7 Frank Y. Shih et al. "Robust watermarkingand compression for medical images based on genetic algorithms" Elsevier, Int' J. Information sciences 175 , 2005, pp 200-216

8 Majid Rabbani and Paul W. Jones, "Image Compression Techniques for Medical Diagnostic Imaging Systems" J of Digital Imaging Vol 4, No 2 May 1991

9 PO-CHYI SU et al., "An Integrated Approach to image watermarking and JPEG-2000 Compression" J of VLSI Signal Processing 27, 35-53, 2001
10 Salih Burak Gokturk et al., “ Medical Image Compression based on Region of Interest, with application to COLON CT Images ", Int' Conf of IEEE engineering in medicine and Biology Science, Oct $25^{\text {th }}-28^{\text {th }} 2001$.

11 Bradley J. Erickson M.D. "Irreversible Compression of medical images" J of Digital Imaging, Vol 15, No 1, March 2002,pp 5-14.

12 Rajeshvaran Logeswaran, "Model -Based Compression for 3D medical images stored in the DICOM format" J of Medical System ,Springer 2006, 30:pp, 133-138

13 Kamrul Hasan TalukderI et al., "Haar Wavelet Based Approach for Image Compression and Quality Assessment of Mathematics", Feb 2007

14 By Lihong ZHAO et al., "Medical image lossless compression based on combining an integer wavelet transform with DPCM", Front. Electr. Electron. ng. China 2009, 4(1): pp 1-4 DOI 10.1007/s11460-009-0014-1, Higher Education Press and Springer-Verlag 2008

15 D.Maheswari et al., "Secure layer Based Compound Image Compression xml Compression", IEEE, 2010.

16 M. P. Bakulina, "Efficient Image Compression by Coding of Low-Entropy Sources" ISSN 8756-6990, Optoelectronics, Instrumentation and Data Processing, 2011, Vol. 47, No. 1, pp. 47-52

17 Nik Shahidah Afifi Bt Md Taujuddin, et al. "Enhancement of medical image compression by using Threshold redicting Wavelet- Based Algorithm" Springer- Verlag Berlin Heidelberg, 2011

18 Sujitha Juliet Devaraj, "3-D Medical Image Compression for Telemedicine Application”, Int' Conf on Modeling, Optimisation and Computing (ICMOC 2012), elsvier,SciVersecience Direct Procedia Engineering 38, pp 1444-1449

19 V.K. Bairagi, et al., "The role of Transforms in Image Compression, Int. J. of Eng. India Ser Springer" June-August 2013, pp 135-140.

20 .Sujitha Juliet et al, “A Novel medical image compression using Ripplet transform”, Int' J. Real- Time Image Proc, $25^{\text {th }}$ july 2013.

21 Abdul Khader Jilani Saudagar et. al. , “ Image Compression approach with ridgelet transformation using modified neuro modeling for biomedical images", Int. J. of Neural computation and Application, 2014, pp. $1725-1734$

22 M .Moorthi, R. Amutha, " An Integrated Model for Compression of Medical Images in Telemedicine", Proc. Elsevier Int. Conf. on Emerging Research in Computing, Information, Communication and Application (ERCICA), 2014.pp 687-694

23 Sujitha Juliet et al., "A novel image compression method for medical images using Geometrical regularity of image structure, springer, SIVip, (2015) 9, pp. 1691-1703

24 26. V. K. Bairagi, "Symmetry Based Biomedical Image Compression" Int. J. Digital Imaging, Springer, 24 Feb, 2015.

25 Sujitha Juliet et al. "Projection - Based Medical Image Compression for Telemedicine Applications", Int' J digit Imaging(2015) 28, pp. 146-159

26 Manpreet Kaur, et al., "ROI Based Medical Image Compression for Telemedicine Application", $4^{\text {th }}$ Int finger Conf on Eco-friendly Computing and communication Systems, ICECCS, 2015, Elsvier, Procedia Computer Science, pp 579-585

27 Emmanuel B.S. et al, "Improved algorithm for biometric fingerprint image compression", American J Computation, communication and Control, 2015, 1(5),pp. 75-85 
28 Gran Badshah et al., "Watermark Compression in medical image watermarking using Lempel-Ziv-Welch(LZW) lossless Compression Technique" J.Digit Imaging, springer $16^{\text {th }}$ Oct 2015

29 MinhN.DoandMartin Vetterliy, "Discrete Ridgelet Transforms for Image representation" https://infoscience.epfl.ch/record/52386/files/IC_TECH_ REPORT 200119.pdf

30 David Taubman, "high performance scalable image compression with EBCOT" 1999 IEEE, pp. 344-348

31 Jeff Harrison, "Testbed-12 Compression Techniques Engineering Report”, 2017-05-15 ,OGC 16-055 ,URL: http://www.opengis.net/doc/PER/t12-A069 Category 